\title{
Networking Challenges and Principles in Diffusion-based Molecular Communication
}

\author{
I. Llatser, A. Cabellos-Aparicio and E. Alarcón
}

\begin{abstract}
Nanotechnology has allowed building nanomachines capable of performing simple tasks, such as sensing, data storage or actuation. Nanonetworks, networks of nanomachines, will allow cooperation and information sharing among them, thereby greatly expanding the applications of nanotechnology in the biomedical, environmental and industrial fields. One of the most promising paradigms to implement nanonetworks is Diffusion-based Molecular Communication (DMC). In DMC, nanomachines transmit information by the emission of molecules which diffuse throughout the medium until they reach their destination. Most of the existing literature in DMC has focused on the analysis of its physical channel. In this work, the key differences of the physical channel of DMC with respect to the wireless electromagnetic channel are reviewed with the purpose of learning how they impact the design of networks using DMC. In particular, we find that the uniqueness of the physical channel of DMC will require revisiting most of the protocols and techniques developed for traditional wireless networks in order to adapt them to DMC networks. Furthermore, guidelines for the design of a novel network architecture for DMC networks, including fundamental aspects such as coding, medium access control, addressing, routing and synchronization, are provided.
\end{abstract}

Index Terms-Networking challenges, Diffusion-based Molecular Communication, Nanonetworks.

\section{INTRODUCTION}

$\mathbf{T}$ $\mathrm{HE}$ recent progress in the field of nanotechnology enables the development of nanomachines, i.e., systems in a scale ranging from one to a few hundreds of nanometers capable of performing simple tasks, such as sensing, computing, data storage or actuation. These nanomachines are not just downscaled versions of macroscale systems, but they show novel behaviors due to the unique properties of nanomaterials and nanodevices. For instance, novel nanosensors are able to detect the presence of viruses and other harmful agents, or to sense chemical compounds in concentrations as low as one molecule.

However, the capabilities of a single nanomachine are very limited, due to its reduced size and tight energy constraints. Nanonetworks, i.e., networks of nanomachines, are envisaged to extend the potential of nanomachines both in terms of complexity and range of operation [1]. For instance, wireless nanosensor networks [2] represent a particular case of nanonetworks in which nanosensor motes communicate among them in order to cover larger areas and reach unprecedented locations.

Numerous applications of nanonetworks have been proposed in the biomedical, environmental and industrial

I. Llatser, A. Cabellos-Aparicio and E. Alarcón are with the NaNoNetworking Center in Catalunya (N3Cat), Universitat Politècnica de Catalunya, Barcelona, Spain. E-mail: \{llatser, acabello\}@ac.upc.edu, eduard.alarcon@upc.edu fields [2]. Biomedical applications show the best potential with respect to the unique characteristics of nanonetworks, since the nanoscale is the natural domain of molecules, proteins and DNA sequences. Moreover, nanomachines may provide an interface between biological phenomena and macroscale electronic systems. For instance, in intra-body networks [3], a group of nanosensors will gather data about the level of different substances or the presence of certain agents (e.g., cancer biomarkers) and will transmit it wirelessly to the macroscale [2]. This way, intra-body networks are envisaged to enable the implementation of novel ultra-accurate health monitoring systems.

How nanomachines will communicate is still an important challenge for the networking community. Several communication paradigms have been proposed to implement communication at the nanoscale. Among them, one of the most promising is Molecular Communication (MC) [4], a biological mechanism found in nature. $\mathrm{MC}$ is used by cells to communicate among them, and it encodes information into molecules that are released by the transmitter and physically transported to the receiver. MC can be classified into three categories, according to how molecules propagate across the medium [5]. First, in walkway-based MC, molecules follow predefined pathways to reach the receiver (e.g., transported by molecular motors). Second, in flow-based MC, molecules are released in a fluid medium and they are guided by currents or flows to their destination, such as in hormonal communication through the blood stream. Last, in diffusionbased MC, molecules are suspended in a fluid medium and they move due to the effect of free diffusion. Diffusion-based Molecular Communication (DMC) encompasses several MC techniques, such as calcium ion signaling, one of the most important communication mechanisms among living cells, and pheromonal communication. For this reason, this paper will focus on DMC, which is also the most widely studied MC technique to date.

Due to its biological nature, DMC is based on different fundamental principles when compared to traditional wireless communications. First and foremost, traditional wireless communications are based on the transmission of continuous electromagnetic (EM) waves, while DMC takes base on encoding the information into a discrete number of molecules. Second, whereas the propagation speed of EM waves has a deterministic and constant value in the order of the speed of light, in DMC the propagation of the molecules across the medium is due to spontaneous diffusion, which is a stochastic process and occurs at a much lower speed, in the order of a few millimeters per second. Third, as opposed to the EM prop- 
agation in the free space, the diffusion process introduces a high amount of distortion in the transmitted signals. Fourth, as we will show next, several key communication metrics of the DMC channel scale differently when compared to traditional wireless communications. As a result of these differences, the design of DMC systems imposes new fundamental challenges to the networking research community. Furthermore, existing communication paradigms, protocols and architectures for wireless communication networks must undergo a profound revision before they can be applied to this new scenario.

In this work, we first review the key differences of the physical channel of DMC with respect to the wireless EM channel. We find that the uniqueness of the physical channel of DMC will require revisiting most of the protocols and techniques developed for traditional wireless networks in order to adapt them to DMC networks. Therefore, we carry a systematic analysis of the networking challenges in DMC and we point out the principles over which DMC networks will take base. Throughout this analysis, we make a significant effort to take very few assumptions with respect to the biological implementation of the system; therefore, both the challenges and the DMC principles described in this paper are as technology-agnostic as possible.

The remainder of this paper is organized as follows. In Section II, we briefly describe DMC and enumerate the assumptions taken in this paper. Section III reviews some results in communication metrics in DMC and compares them to their equivalent in traditional wireless communications. In Section IV, we identify the main aspects that make DMC networks radically different from traditional wireless networks, and what challenges they introduce in the design of protocols and techniques for DMC networks. Finally, Section V concludes the paper.

\section{Diffusion-BASed Molecular COMmunicAtion}

A DMC network consists of a set of nanomachines located in a fluid medium communicate by means of a DMC process. A DMC process is composed of three main phases: emission, propagation and reception. First, transmitter nanomachines encode the information to be sent into the release pattern of molecules. The emitted molecules cause a variation in their local concentration, which then propagates throughout the medium due to a diffusion process. Finally, receivers are able to estimate the concentration of molecules in their neighborhood. This measurement activates specific signal transducing mechanisms which allow the receivers to decode the transmitted information. Figure 1 illustrates a scenario of multipointto-multipoint DMC network with multiple transmitters and receivers, as is envisaged in future nanonetworks.

The integration of the molecular transceivers needed for the emission and reception of information into nanomachines will be inspired on the mechanisms developed by nature for communication among living cells, such as the ligandbinding reception process. Moreover, the use of chemicallydriven transceivers will ensure the bio-compatibility of the communication process, as well as an extremely high energy efficiency [4].

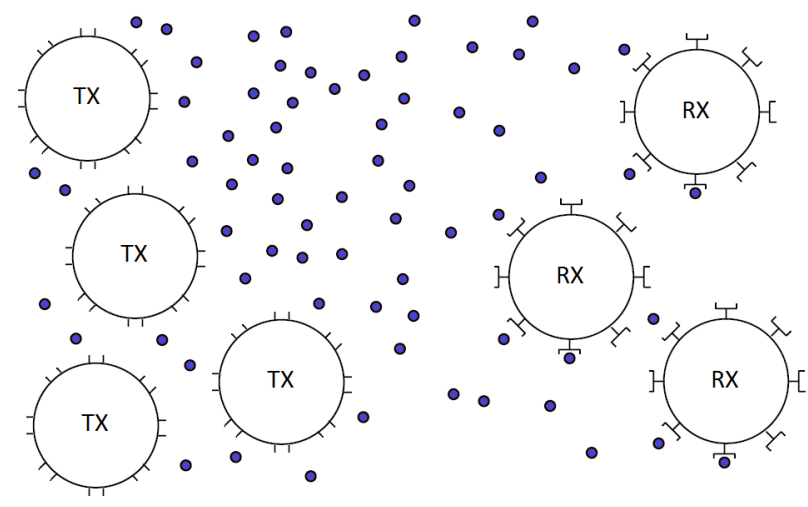

Fig. 1. Schematic diagram of a multipoint-to-multipoint DMC network.

\section{A. Main Assumptions}

In this work, we consider a DMC scenario with the following assumptions:

1) In our envisaged scenario, the concentration of emitted molecules is much lower than the concentration of the fluid molecules. Under this condition, we assume that interactions among the emitted molecules (e.g., collisions and electrostatic forces) can be neglected. An example of this kind of scenario is calcium ion signaling among cells, where extracellular concentration of calcium ions is in the millimolar range, while the concentration of water (the main component of extracellular fluid) is of 55.5 molar, more than 4 orders of magnitude higher. In this scenario, each of the molecules released by a transmitter moves according to Brownian motion, a stochastic process with independent increments which are normally distributed with zero mean. Since the movement of each molecule is independent, molecular diffusion can be modeled by Fick's laws of diffusion with a homogeneous diffusion coefficient both in space and time.

2) Even though nanomachines are expected to have limited memory storage and be capable of performing only simple calculations, we assume them to be able to reproduce the algorithmic behavior of computers and compute the same set of mathematical operations. Indeed, the latest advances in synthetic biology are allowing the development of small biocomputers [6].

3) Motivated by the expectedly low complexity of nanomachines, which prevents the use of advanced modulations, we assume a digital pulse-based modulation to encode the information. Even though most of the results derived in this work apply to any modulation type, this assumption allows the quantitative modeling of signal propagation in DMC networks. According to this scheme, a transmitter nanomachine encodes the information into pulses of molecules, which are instantaneously released to the environment. The release of a molecular pulse creates a delta in the molecular concentration at the transmitter location, and its propagation throughout the environment can be analytically modeled by solving Fick's laws of diffusion. Fig. 2 shows the expected value 


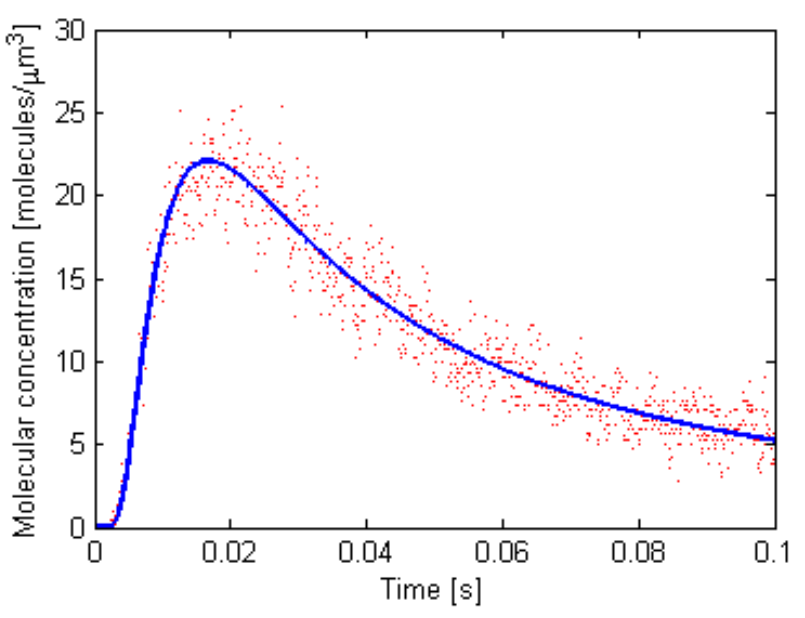

Fig. 2. Molecular concentration at the receiver location as a function of time, for a case example where the number of transmitted molecules is $Q=$ $3 \cdot 10^{5}$, the transmission distance is $r=10 \mu \mathrm{m}$ and the diffusion coefficient is $D=1 \mathrm{~nm}^{2} / \mathrm{ns}$. The blue line shows the analytical value of the concentration, whereas the red dots correspond to the actual measurements by the receiver.

of the molecular concentration at the receiver location as a function of time, after the transmitter has released a pulse of molecules [7] (blue line). The receiver is modeled as a sphere able to measure the concentration of molecules within its boundaries. However, since the molecules move according to Brownian motion, the actual concentration measured by the receiver does not match its expected value, but it is a stochastic process which can be calculated numerically (red dots). We observe that the value of the molecular concentration sharply increases from zero up to its maximum value. After reaching its peak, the concentration slowly decreases, forming a long tail due to the effect of diffusion.

4) We focus on the application scenario of nanonetworks, where nanomachines represent the nodes of the network. Therefore, we consider a general case of multipoint-tomultipoint communication among nanomachines, with multiple transmitters and receivers communicating in a shared medium.

\section{Review of CommunicAtion Metrics in DMC}

In our previous work, we analyzed the scalability of several key communication metrics with respect to the transmission distance, in a DMC scenario with the previous assumptions [7], [8]. Crucially, we concluded that, due to the uniqueness of the physical channel of DMC with respect to that of traditional wireless communications, there exist fundamental differences in the scalability of the communication metrics in both scenarios.

First, we observed that the propagation delay scales as $\Theta\left(r^{2}\right)$. The Big Theta notation means that the average propagation delay is proportional to the square of the transmission distance, even if its actual value will probably differ slightly due to the effect of random diffusion. This result contrasts with the behavior seen in traditional wireless communications, where the delay scales as $\Theta(r)$. The reason for the higher
TABLE I

COMMUNICATION METRICS IN TRADITIONAL WIRELESS COMMUNICATIONS AND DMC. $r$ STANDS FOR THE TRANSMISSION DISTANCE.

\begin{tabular}{l|c|c} 
Metric & Wireless communications & DMC \\
\hline Pulse delay & $\Theta(r)$ & $\Theta\left(r^{2}\right)$ \\
Pulse amplitude & $\Theta\left(1 / r^{2}\right)$ & $\Theta\left(1 / r^{3}\right)$ \\
Pulse energy & $\Theta\left(1 / r^{2}\right)$ & $\Theta(1 / r)$ \\
Pulse width & $\Theta(1)$ & $\Theta\left(r^{2}\right)$ \\
Pulse duration & $\Theta(1)$ & $\Theta\left(r^{2}\right)$
\end{tabular}

delay in DMC is that the transmitted signals do not have a constant propagation speed as in traditional wireless communications, but the propagation of the molecules is governed by the stochastic process of Brownian motion.

We considered two alternatives for the detection of transmitted pulses: amplitude detection [7] and energy detection [8]. Amplitude detection consists in interpreting the received signal as a bit " 1 " if its amplitude (i.e., the maximum concentration) is over a given threshold, and as a bit " 0 " otherwise. On the other hand, using energy detection, the received signal is decoded as a bit " 1 " if its energy is over a given threshold, and as a bit " 0 " otherwise. As opposed to EM communications, where the energy is calculated as the integral of signal power over time, in DMC the pulse energy can be interpreted as the total number of particles received in a time interval known as the pulse duration.

In the case of amplitude detection, receivers detect pulses by measuring their amplitude, which was found to scale as $\Theta\left(1 / r^{3}\right)$. This result indicates that the amplitude of the transmitted signals will decay faster in DMC as compared to traditional wireless communications, where the signal amplitude scales as $\Theta\left(1 / r^{2}\right)$. Moreover, whereas the propagation of EM signals in the free space does not suffer from distortion, in DMC the diffusion process increases the width of the transmitted pulses proportionally to $\Theta\left(r^{2}\right)$. In DMC, the pulse width will determine the minimum interval between transmitted pulses and, ultimately, will limit the maximum achievable bandwidth in a DMC network. Both the faster amplitude decay and the signal distortion observed in DMC are due to the effects of the diffusion process.

In an energy detection scenario, different communication metrics become relevant. Pulses are detected by measuring their energy, which was observed to scale as $\Theta(1 / r)$ [8], in sharp contrast with the scalability of the pulse amplitude. The scalability of the pulse energy in traditional wireless communications is also different; since it is proportional to the pulse amplitude, energy scales as $\Theta\left(1 / r^{2}\right)$. Moreover, the pulse duration, which will be the main constraint on the achievable bandwidth in DMC using energy detection, grows as $\Theta\left(r^{2}\right)$ [8]. As before, the diffusion process causes these metrics to scale worse in DMC with respect to traditional wireless communications.

To summarize, Table I compares the scalability of the communication metrics in DMC and traditional wireless communications. The derivation of these results can be found in our previous work [7], [8]. 


\section{Networking Challenges in Molecular COMMUNICATION}

Based on the differences in the scalability of key communication metrics in DMC with respect to traditional wireless communications found in the previous section, we identify next the main challenges that will appear in the design of DMC networks.

\section{A. Propagation delay}

The rapid increase of the propagation delay as a function of the distance will become a huge limitation in DMC networks. For instance, for transmission distances of just a few tens of micrometers, the delay will be in the order of 1 second (and it will increase with the square of the transmission distance) [7]. This high delay will almost prevent using handshake-based protocols, such as the TCP 3-way handshake, very common in traditional wireless networks. For the same reason, retransmissions will also be very expensive in DMC. Retransmissions, needed when a packet is not correctly received, can be implemented by means of negative acknowledgments. However, in scenarios where the propagation delay is specially high, such as DMC with a relatively long transmission distance, it is possible that even the use of acknowledgments becomes prohibitive. Therefore, the design DMC networks should prioritize maximizing the probability of correctly receiving the transmitted information, thereby minimizing the number of retransmissions needed.

Another particularity of DMC networks related to their expectedly high propagation delay concerns the Medium Access Control (MAC). In traditional wireless networks, the most widespread MAC protocol is CSMA, which is based in sensing the medium in order to verify the absence of traffic before transmitting. However, the high delay in DMC prevents the use of carrier sensing; for instance, at a given time instant, the transmitter may sense a channel free of molecules, but the medium may be busy at the receiver location. An alternative MAC protocol which has been proposed for DMC is Molecular Division Multiple Access (MDMA) [9]. This technique uses different molecule types in order to perform several simultaneous transmissions, sharing the same medium but without interfering each other. Some concerns regarding this technique include the choice of molecule types to be used by different transmitters, so that they do not interact among them and that receivers are able to discern each of the received molecules from one another.

An additional related challenge is user synchronization. Synchronous communication protocols require that the transmitter and receiver are synchronized. However, the long and random propagation delay in DMC makes achieving synchronization among nodes a challenging task. Quorum Sensing, a mechanism used by bacteria and social insects to coordinate their behavior, might also be used to achieve synchronization in a DMC scenario [10]. However, due to the randomness of the diffusion process, there are doubts regarding the level of synchronization that can be achieved with this technique.

\section{B. Channel attenuation}

Another important bottleneck in the performance of DMC networks is the channel attenuation, which will limit the transmission range of nodes in a DMC network. The channel attenuation depends greatly on the method used by the receivers to detect the transmitted pulses of molecules. As we have previously described, the pulse amplitude and the pulse energy scale very differently with respect to the transmission distance: $\Theta\left(1 / r^{3}\right)$ and $\Theta(1 / r)$, respectively. Therefore, in terms of channel attenuation, energy detection seems better suited to a DMC scenario than amplitude detection.

A complementary approach to combat channel attenuation considers using a cooperative approach for the transmission of pulses [11], as opposed to the selfish approach typical in traditional wireless networks. Following this approach, a group of nodes coordinate to simultaneously transmit a molecular pulse. As a result, their individual contributions aggregate into a pulse with a higher amplitude. Of course, this approach requires that the nodes are synchronized (e.g., by means of Quorum Sensing, as previously described). In any case, it should be investigated whether the performance of this new signal amplification technique for DMC outperforms that of protocols widely used in traditional wireless networks, such as multi-hopping.

\section{Channel distortion}

Because of the stochastic nature of Brownian motion, the physical channel of DMC distorts the transmitted pulses. Fig. 2 shows the molecular concentration, as a function of time, measured by a receiver nanomachine after a transmitter releases a spike of molecules. Indeed, as it can be observed, the concentration measured by the receiver no longer resembles a delta, but it has an infinite tail. This huge distortion of the transmitted pulses presents a further challenge to their successful detection and represents the main limitation in the achievable bandwidth in DMC.

From an amplitude detection perspective, the channel distortion causes the transmitted pulses to have a width which increases with the transmission distance. The pulse width will limit the minimum time interval between two consecutive pulses and, therefore, will introduce an upper bound on the achievable communication bandwidth. On the other hand, using energy detection, it is the pulse duration which limits the maximum bandwidth. Even though both the pulse width and the pulse duration increase proportionally to the square of the transmission distance, i.e., $\Theta\left(r^{2}\right)$, the pulse duration was found to be around one order of magnitude higher than the pulse width in a typical scenario [8]. In consequence, and as opposed to what happened with regards to channel attenuation, amplitude detection offers a better performance than energy detection in terms of achievable bandwidth in DMC networks.

Furthermore, the pulse distortion also creates intersymbol interference (ISI). In contrast with EM communications, where the ISI can be greatly mitigated by means of adaptive equalization, in DMC the interference caused by the infinite tails of molecular pulses is hard to avoid. Therefore, novel techniques to minimize the effect of ISI in DMC need to be investigated, 
such as absorbing the molecules from the previous transmissions by the receivers, or using degrading molecules in order to simulate a Time To Live (TTL) mechanism in the emitted molecules.

\section{Limited capabilities of nanomachines}

Due to their reduced size, in the order of a few micrometers, nanomachines are expected to have strict limitations in terms of processing power, memory storage, available energy and number of molecules they can emit. As an example, a memory device with a record storage density of $3.6 \mathrm{Tbit}^{\mathrm{inch}}{ }^{2}$ was recently demonstrated [12]. With this storage density, a memory device with a size of $10 \mu \mathrm{m}^{2}$ (in line with the expected size of the envisaged nanomachines) would have a capacity of just 7 kbytes.

These constraints will not allow the use of computationally complex modulations and protocols, such as the ones used in traditional wireless networks. On the contrary, they represent the main motivation for the use of simple opportunistic techniques, such as the pulse-based modulation assumed in this work. However, we cannot forget that the main drawback of these simple modulations and coding schemes is that they require a higher signal-to-noise ratio at the receiver than more complex techniques.

Furthermore, in order to minimize the number of retransmissions (because of the high propagation delay in DMC), error correcting codes are needed. Given the limitations in terms of processing power of nanomachines, very simple error correcting coding schemes, such as repetition codes, will be most suitable in a DMC scenario.

\section{E. Node mobility}

In a typical scenario of DMC, such as an intra-body network, the network nodes will be suspended in a fluid medium. In this case, their movement can be modeled as Brownian motion, i.e., the node trajectory (as it happened with the emitted molecules) will have an unpredictable pattern. Note that, even though both the emitted molecules and the network nodes move according to Brownian motion, the latter will have a much lower diffusion coefficient due to their larger size, and therefore they will move at a much slower speed.

A paradigm that takes advantage of node mobility to improve the communication performance is that of Delay Tolerant Networks. In these networks, whenever some information is ready to send, it is not immediately transmitted. Instead, the data is stored in memory and the transmission is postponed until the transmitter detects that its location is close enough to the intended recipients. Therefore, transmitters can select their emission time in order to achieve a successful communication.

In order to apply this technique, a mechanism to measure the distance between nanomachines is needed. Besides its other applications, Quorum Sensing could also be used for distance estimation in DMC. Indeed, using Quorum Sensing bacteria are able to estimate their local population by sensing the concentration of signaling molecules known as autoinducers, which are continuously released by the bacteria. Similarly, receiver nanomachines could release a special type of molecules that would allow transmitters to estimate their distance to potential receivers. Another option to estimate the distance between nanomachines would be to measure the round-trip time of a pulse of molecules released by the transmitter [13].

However, as we previously explained, nanomachines might not have enough memory and processing power to implement these techniques. Therefore, it seems difficult to exploit the node mobility to improve the communication performance of DMC networks.

\section{F. High node density}

A characteristic of most DMC networks in realistic scenarios will be a very high number of nodes located in a small region. For instance, for a transmission range of $100 \mu \mathrm{m}$, the number of nodes required to cover a volume of just $1 \mathrm{~cm}^{2}$ is in the order of $10^{6}$.

With such a high number of nodes, and given the limited capabilities of nanomachines, giving a unique address to each network node, as is done in traditional wireless networks, will become nearly impossible. For instance, aspects such as updating the routing tables of nanomachines or assigning a unique address to a new node entering the network, would become very challenging in the constantly-changing scenario of DMC networks. Instead, an alternative approach would be setting the address to identify the nanomachine type (e.g., nanosensor, nanoactuator, etc.), rather than the individual nanomachine. This way, the previous challenges would be greatly simplified.

Moreover, protocols where nodes compete to access a shared medium do not seem applicable in DMC networks, for two main reasons. First, due to the high number of nodes, it is expected that many of them would be simultaneously competing for the channel at any time. Second, due to the high propagation delay, after every transmission the channel would be busy for a long period of time before it can be reused by another transmitter.

A more feasible alternative for dense networks are cooperative protocols, which have already been proposed for dense wireless networks [14]. Another example of a cooperative approach is the already-mentioned Quorum Sensing.

\section{SUMMARY AND CONCLUDING REMARKS}

In this paper, we have considered a DMC network where nodes transmit information using a pulse-based modulation. Based on this scenario, we have described some of the most relevant design challenges and principles that will appear in future DMC networks. In order to make the obtained results general and technology-agnostic, we have taken as few assumptions as possible.

Fundamental differences between of DMC and EM communications, such as the propagation delay, the channel distortion and the node mobility, have been identified. Due to the uniqueness of the physical channel of DMC, a new network architecture needs to be developed for DMC networks. Indeed, we have observed that many of the protocols and techniques used in traditional wireless networks cannot be directly applied to this novel networking paradigm, and we have provided more suitable alternatives for a DMC scenario. These results, which 
TABLE II

MAIN GUIDELINES FOR DMC NETWORKS.

\begin{tabular}{lll}
\hline \hline Limitation & Challenge & Proposed technique \\
\hline Propagation delay & MAC & MDMA \\
Propagation delay & Synchronization & Quorum Sensing \\
Attenuation & Signal detection & Energy detection \\
Distortion & Bandwidth & Amplitude detection \\
Distortion & ISI & TTL / Molecule absorption \\
Node simplicity & Modulation & Pulse-based modulation \\
Node simplicity & Coding & Repetition codes \\
Node density & Addressing & Nanomachine type addressing \\
Attenuation / density & Signal transmission & Cooperative amplification
\end{tabular}

are detailed in Section IV and summarized in Table II, provide useful guidelines for designers of future DMC networks.

\section{ACKNOWLEDGMENT}

This work has been partially supported by the FPU grant of the Spanish Ministry of Education.

\section{REFERENCES}

[1] I. F. Akyildiz, F. Brunetti, and C. Blázquez, "Nanonetworks: A new communication paradigm," Computer Networks, vol. 52, no. 12, pp. 2260-2279, 2008

[2] I. F. Akyildiz and J. M. Jornet, "Electromagnetic wireless nanosensor networks," Nano Communication Networks, vol. 1, no. 1, pp. 3-19, May 2010.

[3] B. Atakan, O. Akan, and S. Balasubramaniam, "Body area nanonetworks with molecular communications in nanomedicine," IEEE Communications Magazine, vol. 50, no. 1, pp. 28-34, 2012.

[4] T. Suda, M. Moore, T. Nakano, R. Egashira, A. Enomoto, S. Hiyama, and Y. Moritani, "Exploratory research on molecular communication between nanomachines," in Genetic and Evolutionary Computation Conference (GECCO), Late Breaking Papers, 2005.

[5] M. Pierobon and I. F. Akyildiz, "A physical end-to-end model for molecular communication in nanonetworks," IEEE Journal on Selected Areas in Communications, vol. 28, no. 4, pp. 602-611, 2010.

[6] R. V. Solé and J. Macia, "Synthetic biocomputation: The possible and the actual," in European Conference on Artificial Life (ECAL), no. 7 , 2011, pp. 29-36.

[7] I. Llatser, E. Alarcón, and M. Pierobon, "Diffusion-based Channel Characterization in Molecular Nanonetworks," in IEEE International Workshop on Molecular and Nanoscale Communications (MoNaCom), 2011, pp. 467-472.

[8] I. Llatser, A. Cabellos-Aparicio, E. Alarcón, and M. Pierobon, "Performance Evaluation of Energy Detection in Diffusion-based Molecular Communication," submitted for publication.

[9] L. Parcerisa and I. F. Akyildiz, "Molecular communication options for long range nanonetworks," Computer Networks, vol. 53, no. 16, pp. 2753-2766, 2009.

[10] S. Abadal and I. F. Akyildiz, "Bio-Inspired Synchronization for Nanocommunication Networks," in IEEE GLOBECOM, 2011, pp. 53755379.

[11] S. Abadal, I. Llatser, E. Alarcón, and A. Cabellos-Aparicio, "Cooperative Signal Amplification for Molecular Communication in Nanonetworks," in IEEE International Workshop on Molecular and Nanoscale Communications (MoNaCom), 2012.

[12] N. Tayebi, Y. Zhang, R. Chen, Q. Tran, R. Chen, Y. Nishi, Q. Ma, and V. Rao, "An Ultraclean Tip-Wear Reduction Scheme for Ultrahigh Density Scanning Probe-Based Data Storage," ACS Nano, vol. 4, no. 10, pp. 5713-5720, 2010

[13] M. J. Moore, T. Nakano, A. Enomoto, and T. Suda, "Measuring Distance From Single Spike Feedback Signals in Molecular Communication," IEEE Transactions on Signal Processing, vol. 60, no. 7, pp. 3576-3587, 2012.

[14] C. Cetinkaya and F. Orsun, "Cooperative medium access protocol for dense wireless networks," in The Third Annual Mediterranean Ad Hoc Networking Workshop, 2004, pp. 197-207.

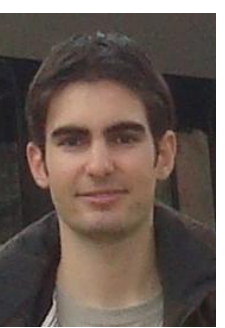

Ignacio Llatser was born in Vinaròs (Spain) in 1984. In 2008, he graduated with a double M.S. degree in Telecommunication Engineering and Computer Science from the Technical University of Catalonia (UPC). He completed his Master Thesis on game-theoretical protocols for vehicular networks in the Laboratory for Computer Communications and Applications, at the École Polytechnique Fédérale de Lausanne (EPFL). In 2009, he joined the Nanonetworking Center in Catalunya (N3Cat) at UPC, where he is currently pursuing a $\mathrm{PhD}$ in Computer Architecture. His research interests lie in the fields of nanonetworks, molecular communication and graphene-enabled wireless communications.

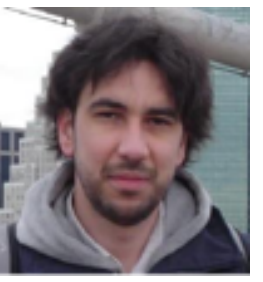

Albert Cabellos-Aparicio received a BSc (2001), MSc (2005) and PhD (2008) degree in Computer Science Engineering from the Technical University of Catalonia. In 2002 he joined the Advanced Broadband Communications Center (CCABA) where he worked as research assistant. In 2004 he was awarded with a full scholarship to carry out $\mathrm{PhD}$ studies at the Department of Computer Architecture, Technical University of Catalonia (UPC), Spain. In September 2005 he became an assistant professor of the Computer Architecture Department and as a researcher in the Broadband Communications Group. In 2010 he joined the NaNoNetworking Center in Catalunya. He is an editor of the Elsevier Journal on Nano Communication Networks and member of the Project Management Committee of the LISPmob open-source initiative. His main research interests are new architectures for the Internet and Nano-networks.

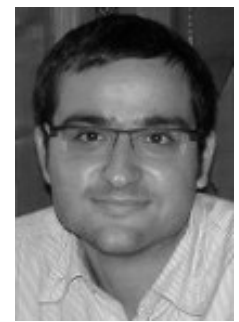

Eduard Alarcón (S'96, M’01), received M. Sc. (national award) and Ph.D. degrees in EE from UPC Barcelona, Spain, in 1995 and 2000, respectively, where he became Associate Professor in 2001, Associate Dean of International Affairs, School of Electrical Engineering, UPC, 2006-09, and visiting Professor at University of Colorado at Boulder, USA (2003). He has coauthored more than 150 international scientific publications, 3 book chapters and 3 patents, and been involved in different national and US R\&D projects. Research interests include the areas of mixed-signal integrated circuits, on-chip energy management circuits, wireless energy transfer and communications at the nanoscale. He has given 17 invited or plenary lectures and tutorials in Europe, America and Asia, and been elected IEEE CAS society distinguished lecturer. He is an elected member of the IEEE CAS Board of Governors (2010-2013), recipient of Best paper award at IEEEMWSCAS98, co-editor of 2 journals special issues, 3 conference special sessions, TPC co-chair and TPC member of several IEEE conferences, and Associate Editor for IEEE TCAS. 